\title{
La coopération militaire belgo- française : oscillations politiques et identitaires
}

\author{
André Dumoulin
}

\begin{abstract}
Attaché à l'Institut royal supérieur de défense (IRSD). Professeur à l'Université de Liège *.
\end{abstract}

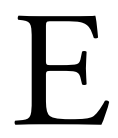

n Belgique, la France est vue comme un allié privilégié. Le Royaume fait partie des intérêts vitaux hexagonaux au sens politique et territorial, et par proximité géographique et culturelle. Malgré certains contentieux africains historiques, la France et la Belgique sont souvent complices concernant la politique de défense (vision d'ensemble, soutien à des dossiers spécifiques de la PESD/ PSDC, Groupe des Quatre, aspects Défense du Traité constitutionnel puis de Lisbonne, QG européen, GT1500, Initiative européenne d'intervention ${ }^{(1)}$ sous l'impulsion du président de la République) ou lors des grands débats (coopération structurée permanente, Livre blanc européen, Stratégie européenne de sécurité, lutte contre le radicalisme ${ }^{(2)}$ ), même si les lignes de conduite ne sont pas toujours symétriques. Vu sa taille et ses intérêts stratégiques, la France a toujours été moins fédéraliste que la Belgique.

Du point de vue opérationnel, le faible poids capacitaire de la Belgique conduit à un accompagnement de la France à la carte, pour des opérations en coalition ou dans le cadre d'alliances. Cet appui dépend en fait de la composition idéologique et du degré d'équilibre linguistique des gouvernements belges incontournablement en coalition avec une vision différenciée du degré acceptable de prise de risque. Mais les accords bilatéraux de coopération militaire avec la France intègrent plus d'une centaine d'activités par an dont des entraînements navals, terrestres ${ }^{(3)}$ et aériens ${ }^{(4)}$ ou des actions communes possibles telles la récupération de ressortissants, le renseignement ou les forces spéciales. En outre, de nombreux stagiaires belges ${ }^{(5)}$ suivent une formation et un entraînement au sein des unités et

\footnotetext{
* N'engage pas les institutions de référence.

(1) Réunions semestrielles des Neuf à Balard. Échanges sur la perception stratégique nationale et les menaces, planification commune à moyen terme, facilitateur d'un engagement plus rapide et volontaire.

(2) Cf. les Accords de coopération franco-belge pour la lutte contre le radicalisme violent.

(3) Par exemples, entraînement (février 2018) de snipers français du 35 RI (Belfort) et leurs homologues du bataillon des Chasseurs ardennais à Marche-en-Famenne et à Elsenborn (Ardennes belges). Exercice Winter Training Vertigo en Autriche (mars-avril 2017) du 12/13 e de Ligne avec le $1^{\text {er }}$ régiment de Tirailleurs français. Exercice belge au Camp français de la Courtine (mars 2017).

(4) Ainsi, deux pilotes français ont été affectés au $2^{\mathrm{e}}$ Wing tactique belges (Florennes) depuis mars 2016. Dans le cadre de l'European Air Group, entraînement mutuel belgo-français depuis la base de Mont-de-Marsan (décembre 2015).

(5) www.ambafrance.org.
} 
écoles françaises alors que des Belges contribuent à l'état-major du Corps de réaction rapide-France (Lille) et travaillent depuis longtemps à l'Eurocorps ${ }^{(6)}$.

Cet accompagnement prudent varie selon les circonstances, les intérêts, la faisabilité, la cohérence, l'efficacité, la plus-value ou le degré de connivencedivergence des partis politiques inscrits dans les exécutifs belges ${ }^{(7)}$. Si en France les morts pour la patrie sont assumés par le poids de la centralité hexagonale, d'un mono-pouvoir politique gouvernemental, du rôle de l'Élysée à la pointe de la " pyramide " et d'une culture stratégique historique assumée, il n'en est pas de même en Belgique traversée par des contingences politiques et communautaires où l'esprit de défense n'est pas assumé en profondeur ${ }^{(8)}$. À Bruxelles, les conséquences de possibles drames à forte létalité seraient très certainement la chute du gouvernement !

Néanmoins, Bruxelles a pris fait et cause pour la demande française d'activer l'article 42.7 du TUE après les attentats de Paris. Du point de vue industriel, des accords de coopération existent en matière technologique et d'achats d'équipements, la Belgique ayant des niches capacitaires à faire valoir avec le soutien du Belgian Security \& Defence Industry (BSDI). La récente décision belge d'acquérir de nouveaux équipements majeurs d'ici 2030 à hauteur de 9,2 milliards d'euros ("Vision stratégique ») (9) pourrait aboutir à de nouvelles coopérations industrielles : remplacement des frégates, des chasseurs de mines et des F-16, achat de drones, programme français Scorpion... L'année 2018 est considérée par le chef de la Défense, le général Marc Compernol, comme une année de vérité.

Déjà, un $M o U$ (Memorandum of Understanding) a été signé entre la Belgique et la France pour l'acquisition ${ }^{(10)}$ confirmée par le ministre de la Défense, Steven Vandeput (N-VA) en juin dernier, pour la composante Terre de l'armée belge, demanderesse de cette coopération franco-belge depuis avril 2017, de 60 véhicules de combat médians de type Jaguar et de 417 véhicules de combat léger de type Griffon autour du programme français Scorpion ; cela en remplacement des véhicules de type AIV Piranha et MPPV Dingo entre 2025 et 2030. Au-delà, il s'agit pour la Belgique d'intégrer la vision française d'utilisation desdits véhicules du point de vue doctrinal mais aussi les concepts de formation, d'entraînement, de communication, de capacité logistique et d'infrastructure, y compris la coopération en milieu urbain, via un exercice à Sissonne au Centre d'entraînement aux actions

(6) Cf. André Dumoulin : «L'Eurocorps : socle d'une défense européenne intégrée ? ", Sécurité \& Stratégie, n 136, IRSD, avril 2018.

(7) Cf. Vincent Moyse et André Dumoulin : "Le processus décisionnel belge en matière d'opérations civilo-militaires ", Courrier hebdomadaire, $\mathrm{n}^{\circ}$ 2086-2087, CRISP, Bruxelles, 2011.

(8) André Dumoulin : "Défense citoyenne et citoyens de la défense : l'armée belge et la nation ", Sécurité \& Stratégie, $\mathrm{n}^{\circ} 130$, IRSD, septembre 2017.

(9) André Dumoulin : "Belgique : vers un rééquipement des forces armées », Tribune $\mathrm{n}^{\circ} 731, R D N, 7$ janvier 2016, Agence Belga, Bruxelles, 30 juin 2016 ; André Dumoulin : «La défense belge du futur », RDN, avril 2015.

(10) Contrat de 1,1 milliard d'euros, moyens de communication et pièces de rechange incluses. 
en zone urbaine (Cenzub) ${ }^{(11)}$ impliquant les $12 / 13^{\text {e }}$ de Ligne belge (101 militaires) et le $1^{\text {er }}$ RTir (150 militaires) sous la forme d'un sous-groupement tactique interarmes. À partir de la signature d'une lettre d'intention signée le 5 avril 2017 entre les chefs d'état-major de l'armée de terre des deux pays, nous voyons naître un partenariat "stratégique majeur » d'autant que cela concerne la Brigade médiane belge et la $7^{\mathrm{e}}$ brigade blindée française ${ }^{(12)}$ qui furent d'ailleurs mises en évidence lors du défilé de la fête nationale du 21 juillet 2017 à Bruxelles.

En outre, il existe un accord de coopération pour la surveillance de l'espace aérien entre le Benelux et la France à propos de menaces aériennes non militaires, dans un processus diplomatique lancé en février $2017^{(13)}$ et approuvé par le gouvernement le 19 octobre de la même année ; intégration des Belges dans le programme de la composante spatiale optique (CSO) du programme MUSIS (Multinational Space-based Imaging System) succédant à Hélios 2 ; accords d'entretien du navire belge Godetia et de maintenance corrective et préventive en condition opérationnelle des 11 chasseurs de mines tripartites belges (et hollandais) entre 2018 et 2021 par la firme française CNN-MCO et... achat annuel par la Défense belge de 50000 rations de combat individuelles réchauffables françaises au prix unitaire de 10 euros.

La coopération belgo-française semble évidente au vu de la proximité géographique et d'une histoire " commune " pour le meilleur ou pour le pire au vu des espaces d'agression du passé (1914-1918, 1940-1945) y compris même durant la guerre froide où dans les plans de frappes de semonce, la province du Luxembourg belge pouvait aussi être ciblée par les Pluton nucléaires tactiques français en cas de débordement du Pacte de Varsovie par le nord-est (effet Sedan). En vérité, il faut nécessairement replacer l'histoire belge dans une perspective d'influence, d'instrumentalisation et de pénétration française dans le Royaume de Belgique (économie, rachat, par défaut d'une solution belgo-belge, de la Générale de Belgique par Suez et de Fortis par BNP Paribas...). Outre le fait que la Belgique est dix-huit fois plus petite en surface géographique que l'Hexagone. Les caractéristiques spécifiques des deux États (République/Royaume), les aspects communautaires belges, les conceptions différentes sur le fédéralisme européen et sur la supranationalité peuvent en grande partie expliquer ces escarmouches entre voisins. La différence de taille, d'ambition et d'histoire a amené divergences et rivalités, sans que ceux-ci n’aboutissent à des ruptures majeures ${ }^{(14)}$ quand bien même dans le folklore flamand, on fête chaque 11 juillet depuis quarante ans, date choisie en référence à la victoire à

(11) Permet, entre autres, de travailler les TTPs (Tactics, Techniques, and Procedures) ainsi que les MOUT (Military Operations on Urbanized Terrain).

(12) Cinq unités belges sont jumelées avec cinq régiments français : le $12^{\mathrm{e}}$ de Ligne Prince Léopold - $13^{\mathrm{e}}$ de Ligne est jumelé avec le $1^{\text {er }}$ régiment de tirailleurs ; les Carabiniers Prince Baudouin - Grenadiers est jumelé avec le $152^{\mathrm{e}}$ régiment d'infanterie ; le Bataillon Libération $-5^{\mathrm{e}}$ de Ligne est jumelé avec le $1^{\text {er }}$ régiment de chasseurs ; le Bataillon des Chasseurs Ardennais est jumelé avec le $35^{\mathrm{c}}$ régiment d'infanterie ; le 1/3 Bataillon de Lanciers est jumelé avec le $5^{\mathrm{c}}$ régiment de dragons (www.defense.gouv.fr/).

(13) À l'Avant-Garde, 19 avril 2018 (www.defencebelgium.com).

(14) Cf. Romain Yakemtchouk : La Belgique et la France. Amitiés et rivalités; L'Harmattan, Paris, 2010. 
Courtrai des milices flamandes et namuroises face à la cavalerie française en... 1302 ! Reste que pour Béatrice Delvaux, éditorialiste au quotidien Le Soir en date du 28 juin 2018 : "La Flandre nourrit un rejet quasi instinctif de ce qu'elle considère comme l'impérialisme atavique français. " Et si à Liège on fête le 14 juillet dans les rues, le rattachisme n'intéresse qu'une toute petite minorité de francophones.

De la même manière qu'avec les programmes de coopération beneluxiens (vu comme laboratoire d'intégration autant que faire se peut) ${ }^{(15)}$, le pragmatisme et la modestie belge restent de mise. L'idée est de coopérer autant que possible (multinationalisation quasi-complète des forces armées) et d'éviter de "mettre toutes ses billes dans le même panier ». Toutes ces orientations vont dans le même sens - avec plus ou moins de bonheur et d'efficacité dans le message délivré et l'application ainsi faite de l'institution " armée belge » - à savoir : alléger pour muscler, simplifier pour crédibiliser, moderniser pour " proactiver ", coopérer et européaniser pour solidariser.

Reste que la dimension politique belge est prégnante à tous les étages et elle n'est pas simple à appréhender et à influencer pour l'esprit cartésien français. Le Royaume de Belgique est traversé par des courants idéologiques, politiques, linguistiques, économiques, culturels à la fois interactifs autant que divergents. États relationnels fluctuants avec certaines constantes. Ce sont les partis politiques ${ }^{(16)}$ qui donnent encore le ton des gouvernements de coalition même si les développements récents en Europe - méfiances populistes, affairisme politiques - rendent les partis moins attrayants pour les citoyens et dès lors plus prudents dans leurs stratégies. Il n'en demeure pas moins vrai qu'en Belgique les partis politiques dominent encore la scène décisionnelle et médiatique.

Cela se reflète idéologiquement au sein des cabinets ministériels (relais des partis) ${ }^{(17)}$ dont aussi celui de la Défense. Jeu d'influence, caisse de résonance, lieu de confrontation idéologique, les cabinets sont le reflet aussi d'orientations spécifiques que certains étiquettent comme tels : budgétaires, communautaires et idéologiques (ministre Delcroix), conceptuelles et budgétaires (Poncelet), qualitatives, éthiques et européistes (Flahaut), euro-atlantistes (De Crem), nationalistes/ régionalistes et euro-atlantistes (Vandeput). Les clivages sont bel et bien présents, atténués par la politique du compromis quand cela est possible mais les phénomènes de paralysies ou de crises organisées pour faire chuter des gouvernements dans des moments favorables pré-électoralement (" débrancher la prise ») existent bel et bien.

(15) Relevons la signature, en juin 2018, de deux $M o U$ entre la Belgique et les Pays-Bas pour l'acquisition totale de 4 frégates et de 6 navires de lutte contre les mines (2023-2030), Bruxelles s'octroyant 2 frégates et 5 chasseurs de mines. Cf. aussi Nicholas Fiorenza : "Briefing Benelux armed forces ", Jane’s Defence Weekly, 23 mai 2018.

(16) Wilfried Dewachter et Sam Depauw : Bureau de partis, bureaux du pouvoir ; Éditions de l'Université de Bruxelles, 2007.

(17) Cf. Les cabinets ministériels et autres. Statuts, rôles et pouvoirs, Bruylant, Bruxelles, 2001 ; Alain Eraly : Le pouvoir enchaîné. Etre ministre en Belgique, Labor, 2002. 
Mais la polarisation philosophique s'est en partie déplacée vers la polarisation régionaliste et socio-économique (Pascal Delwit, 2011) ce qui rend les dossiers économiques en matière de défense très délicats, sachant par ailleurs qu'en "Belgique tout est dans tout » et que l'on négocie dans un même paquet de " donnantdonnant " des dossiers divers et variés qui peuvent ne pas être uniquement dans le domaine de la défense ! Aussi, il n'est pas impossible que les signatures formelles et définitives de l'achat des véhicules terrestres, des drones, des frégates et des chasseurs de mines forment un seul paquet à finaliser à l'automne (juste avant ou juste après les élections municipales d'octobre 2018), avant les élections fédérales de mai 2019 ou à " refiler " à une prochaine coalition (horizon glissant).

Ces quelques grands facteurs (permanence des alliances, prégnance des coalitions politiques, différentiel de mentalité ethnolinguistique et communautaire, degré de priorité socio-économique) caractérisent la politique de défense du pays. Les socialistes et les sociaux-chrétiens francophones, stimulés par l'ouvrage du commandant Gennart, ancien commandant de la base de Florennes (devenu Échevin MR à Namur), mettent régulièrement en avant « la flamandisation " de la Belgique ${ }^{(18)}$ dont l'armée, tandis que le Premier ministre libéral francophone assura plusieurs fois tenir compte des équilibres régionaux. Depuis deux ans, des escarmouches par médias interposés opposent les nationalistes flamands de la N-VA et certains partis politiques francophones dans l'opposition, sachant que pour des raisons démographiques, la Flandre majoritaire contrôle davantage les décisions parlementaires fédérales.

Et c'est là qu'intervient le dossier du remplacement des 54 F-16 belges. Vaste foire d'empoigne, véritable sac de nœuds aux multiples rebondissements budgétaires, techniques, communautaires et parlementaires que cette saga ${ }^{(19)}$ qui concentre toutes les caractéristiques du processus de décision complexe à la belge. De toute évidence, Paris n'a pas vu venir cette complexification accentuée par le déséquilibre politique issu des élections fédérales passées.

Depuis 2016, les lobbies industriels dans leurs réunions d'information autant que les «persuasions » diplomatiques furent légions. Une " guerre des argumentaires " alla crescendo, relayée avec plus ou moins de précision et d'indépendance par les médias, les revues spécialisées et différents blogs. Début mars 2017, le processus de remplacement des F-16 était enclenché, le ministre de la Défense présentant à huis clos le cahier des charges en Commission des achats militaires de la Chambre des Représentants (Parlement). Au final, restèrent en ligne le F-35 américain et l'Eurofighter. Le Rafale F3R concourra finalement hors du schéma imposé

(18) Cf. Le dossier sur le sujet de l'hebdomadaire Le ViflL'Express, 9 décembre 2016.

(19) Pour davantage de détails, cf. les réunions des commissions de la défense de la Chambre des Représentants de Belgique des années 2017 et 2018 ; André Dumoulin, "La saga du remplacement des F-16 belges : un véritable sac de nœuds politico-militaire, Revue militaire suisse, T1, 2018, p. 16-19; Wally Struys, "Les retombées sociétales de l'acquisition d'un avion de combat. Un long fleuve belge... turbulent ", Revue militaire suisse, T1, 2018, p. 20-25. 
par la Belgique en proposant plutôt un cadre stratégique global et intégrateur de technologie, dès novembre 2017.

Sans refaire l'histoire de ces jeux d'influence, il semble bien que dans les interactions belgo-françaises, la méfiance a prévalu. Pour Dassault, il s'est agi de considérer que l'appel d'offres officiel belge (Request for Government Proposal) lancé en mars 2017 était par trop calibré ${ }^{(20)}$ et orienté au bénéfice du choix américain du F-35. Pour l'État français partenaire premier pour l'achat " de gouvernement à gouvernement " il devenait impossible de prendre le risque d'entrer dans la procédure officielle belge mais de choisir - plutôt de proposer - un partenariat approfondi, structurant, privilégié et bilatéral associant l'achat de 34 Rafale (dont certains aéronavalisables) par la Belgique et le suivi technologique des versions futures de l'appareil qui doit bénéficier également des avancées technologiques des recherches des bureaux d'études autour du système de combat aérien du futur (Scaf). Dans ce dernier cadre, Bruxelles a été aussi proposé comme partenaire industriel prioritaire pour négocier des retombées technologiques et économiques. Paris n'a pu aller très loin dans les détails dès lors qu'elle demandait à la Belgique de signer un accord de non-divulgation, notamment avec le constructeur Dassault, de certaines informations précises. Ce que Bruxelles refusait encore en ce début juillet estimant que Paris n'avait pas joué le jeu en ne participant pas à l'appel d'offres public quand bien même la proposition française s'inscrit dans la perspective d'une politique de défense européenne, orientation soutenue par les AE belges. Début juillet 2018, la Belgique fit analyser juridiquement cette demande française.

Au final, et sauf retournement de situation ou choix rapide, le gouvernement belge va, suite à la demande expresse de son Premier ministre, Charles Michel (libéral francophone), analyser l'éventuelle prolongation des F-16, l'analyse de la proposition française et les deux offres reçues des Britanniques et des États-Unis (21), avec, à la clef, éventuellement, de nouvelles empoignades entre la N-VA néerlandophone et le MR francophone, tous deux dans la majorité gouvernementale.

Ainsi, la coopération belgo-française est fortement dépendante des questions politiques internes à la Belgique, des oscillations beneluxiennes, allemandes, britanniques et américaines aux tropismes historiques mais aussi des tensions communautaires et identitaires entre les autorités politiques néerlandophones et francophones ; les premiers sous l'influence de la N-VA ayant un tropisme anglophile et transatlantique, les seconds étant davantage portés - mais pas exclusivement - à soutenir les

(20) À savoir des estimations belges uniquement en dollars, un calendrier d'acquisition conforme au cycle de livraison et calendrier de la seule production américaine, la mise en évidence de la furtivité quand bien même les experts aéronautiques ont constaté une moindre capacité d'emport armement (car soute), une signature infrarouge trop forte et une manœuvrabilité relative. A contrario, les travaux sur le Scaf dans son volet avion de combat semble s'orienter, sur le papier, et en toute incertitude encore vers... de la furtivité, de l'intelligence artificielle, de la connectivité, de la fusion de données (réseau) et de la gestion du champ de bataille (Combat Cloud) : caractéristiques en partie introduites dans le F-35 aux potentialités contradictoires.

(21) Relevons que l'offre américaine et le coût courent jusqu'au mois d'octobre 2018 et l'offre pour l'Eurofighter jusque début 2019. Mais ces offres peuvent être d'évidence reformulées en cas de retard de décision. 
liens avec la France. Nonobstant, nous avons vécu le 19 juillet un psychodrame avec les critiques du Premier ministre belge envers l'ambassadrice française en poste à Bruxelles, $\mathrm{M}^{\mathrm{me}}$ Claude-France Arnould, mécontent de l'immixtion française dans les affaires de l'État belge. En effet, elle avait laissé entendre qu'il ne serait plus possible pour la Belgique de partager le projet Scaf si Bruxelles se prononçait en faveur du F-35. Le choix d'un nouveau chasseur américain ou français dans le remplacement des F-16 belges cristallise les tensions et les orientations géopolitiques internes depuis maintenant deux ans, avec la complexité supplémentaire d'intégrer le volet Trump symbolisé par la guerre commerciale mais aussi certaines indélicatesses possibles dans le pire des scénarios : "Les capacités de Lockheed Martin d'effectuer à distance les mises à jour des logiciels du F-35 » avec, en cas de désaccords diplomatiques, la remise en cause des capacités opérationnelles et logistiques de l'appareil, et, au pire, leur envol (22).

Dans tous les cas de figure, la symbolique du choix par le gouvernement belge tout entier du futur remplacement du Lockheed Martin F-16 à probablement entériner au second semestre 2018, aura des répercussions diplomatiques et industrielles dont on ne connaît pas précisément tous les effets sur le long terme. Assurément, cela laissera des traces, quel que soit le choix final. 